\title{
Critical conditions in complicated patients with oncohematological diseases
}

\author{
Alfia F. Baibulatova, Bulat A. Bakirov \\ Bashkirian State Medical University, Ufa, Russian Federation \\ Contact: Dr. Alfia F. Baibulatova \\ E-mail: baf87@inbox.ru
}

\section{Introduction}

The aim of this study was to assess a direct connection between the development of multiple organ failure syndrome with duration of treatment and outcomes of cytostatic chemotherapy.

\section{Patients and Methods}

We performed a retrospective analysis of 138 adult patients with oncohematological disorders treated with different chemotherapy regimens, followed by admittance to intensive care unit (ICU). The data analysis was performed by assessing functional state of patient's organs and systems using a SOFA scale for evaluation of critical states.

\section{Results}

Significant coagulopathies were diagnosed in more than 120 patients (86.9\% of total group); acute respiratory failure, in 43 cases (31.2\%); impaired consciousness, 9 (6.5\%); acute renal failure, $25(18,1 \%)$; cardiovascular disorders, 58 (42.0\%); acute liver failure, $34(24.6 \%)$. It is also noteworthy that the multiple organ dysfunction occurred in 68 (49.3\%) 


\title{
Критические состояния на фоне терапии у пациентов с онкогематологическими заболеваниями
}

\author{
Альфия Ф. Байбулатова, Булат А. Бакиров \\ Башкирский государственный медицинский университет, Уфа, Российская Федерация
}

\section{Введение и цель работы}

В течение последнего десятилетия достигнуты значительные успехи в изучении патофизиологических изменений в организме при развитии критических состояний. Целью исследования явилась оценка связей между развитием синдрома полиорганной недостаточности, длительностью лечения и исходами цитостатической химиотерапии.

\section{Пациенты и методы}

Нами был проведен ретроспективный анализ 138 взрослых пациентов с онкогематологическими заболеваниями, получающих различные схемы химиотерапии с последующим переводом в блок интенсивной терапии. Анализ был проведен с оценкой функционального состояния органов и систем с применением шкалы критических состояний SOFA.

\section{Результаты}

Более чем у $120(86,9 \%)$ из 138 больных диагностировалась коагулопатия, острая дыхательная недостаточность - у 43 (31,2\%); нарушения уровня сознания - у 9 $(6,5 \%)$; острая почечная недостаточность - 25 (18,1\%); нарушения со стороны сердечно - сосудистой системы - 58 (42,0\%); острая печеночная недостаточность - 34 $(24,6 \%)$. Также обращает на себя внимание, что поли- органная недостаточность развилась у 68 (49,3\%) больных, а сепсис у $24(17,4 \%)$ больных. У 78 больных $(56,6 \%)$ после проведенной полихимиотерапии развилась полиорганная недостаточность. По исходам пребывания в блоке интенсивной терапии 91 пациент $(65,9 \%)$ был переведен в удовлетворительном состоянии в соматическое отделение, а у 47 пациентов констатирован летальный исход (34,1\%). Оценку тяжести органных нарушений у каждого пациента количественно и в динамике проводили с использованием шкалы SOFA. Из обследованных больных умерли 47 пациентов $(34,1 \%)$, от рефрактерных к терапии осложнений, состояние ко-

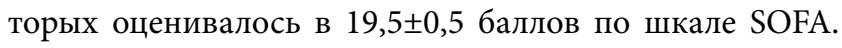
Степень органных нарушений по шкале SOFA оценена в 3-5 баллов у 91 больного, и в 20 баллов - у 47 больных. У пациентов преобладали нарушения со стороны сердечно - сосудистой системы и системы гемостаза.

\section{Заключение}

На основании полученных данных можно судить о возможном развитии летального исхода пациентов онкогематологического профиля, переведенных в блок интенсивной терапии при развитии полиорганной недостаточности.

\section{Ключевые слова}

Онкогематология, химиотерапия, выживаемость, полиорганная недостаточность, интенсивная терапия. 